\title{
Adaptive Pilot-Embedded Data-Bearing Approach Channel Estimation in Space-Frequency Coded MIMO-OFDM Systems
}

\author{
Chaiyod Pirak ${ }^{\dagger *}$, Z. Jane Wang ${ }^{\ddagger}$, K. J. Ray Liu ${ }^{\dagger}$, Somchai Jitapunkul* \\ ${ }^{\dagger}$ Department of Electrical \& Computer Engineering, University of Maryland, College Park, USA. \\ ${ }^{\ddagger}$ Department of Electrical \& Computer Engineering, University of British Columbia, Vancouver, Canada \\ ${ }^{*}$ Department of Electrical Engineering, Chulalongkorn University, Bangkok, Thailand \\ Email: chaiyod.p@gmail.com, zjanew@ece.ubc.ca, kjrliu@eng.umd.edu, somchai.j@ chula.ac.th
}

\begin{abstract}
In this paper, we study the effects of model mismatch error inherently in the FFT-based channel estimation approach when considering the multipath delay profiles with non-integer delays. We propose an adaptive FFT-based channel estimator that employs the optimum number of significant taps such that the average total energy of the channels dissipating in each tap is completely captured in order to compensate the model mismatch error. Furthermore, our proposed pilot-embedded databearing approach is employed for joint channel estimation and data detection. Simulation results reveal that the adaptive FFTbased channel estimator is superior to the FFT-based and LS channel estimators; however, in nonquasi-static fading channels under high Doppler's shift regimes, the performances of all three channel estimators are quite close resulting from the influence of the channel mismatch error that dominates all factors causing the detection error.
\end{abstract}

\section{INTRODUCTION}

High speed data transmission services have been highly demanded in future wireless communications [1]. One promising transmission technique realizing this demand is an orthogonal frequency division multiplexing (OFDM) technique [2]. Recently, multiple-input multiple-output (MIMO) OFDM systems have been proposed for increasing the communication capacity as well as the reliability of the wireless communication systems by exploiting the transmitter and receiver diversities [2]. Beside this promising communication scheme, the space-frequency (SF) coding for MIMO-OFDM systems have been developed for high data-rate wireless communications by exploiting both the spatial and frequency diversities [3]. However, those aforementioned schemes need accurate channel state information (CSI) for coherently decoding the transmitted data, e.g. a maximumlikelihood (ML) decoder. Therefore, channel estimation is of critical importance.

Various channel estimation schemes have been recently proposed for MIMO-OFDM systems [4]-[6]. In [4], a linear minimum mean-squared error (LMMSE) channel estimator was proposed, in which a singular-value decomposition (SVD) is used to simplify the ordinary LMMSE channel estimator. Despite the highly accurate channel estimate of this scheme, it requires

\footnotetext{
${ }^{1}$ This work was partially supported by a Ph.D. scholarship from Commission on Higher Education, Ministry of Education, Thai Government and a grant from the Cooperation Project between Department of Electrical Engineering and Private Sector for Research and Development, Chulalongkorn University, Thailand.
}

intensive computational complexity and the knowledge of the channel correlation. In [5], the FFT-based channel estimation using a certain number of significant taps for estimating the channel impulse response in a temporal domain was proposed. Despite the efficient computational complexity of this scheme, it suffers from an error floor caused by a non-integer multipath delay spread in the wireless channels, known as a model mismatch error. The main contributions of this paper are as follows.

- We study the underlying problem of the FFT-based channel estimator, and solve this problem by proposing an adaptive FFT-based channel estimation that employs the optimum number of significant taps such that the average total energy of the channels dissipating in each tap is completely captured in order to compensate the model mismatch error.

- We also extend the pilot-embedded data-bearing approach for joint channel estimation and data detection in the MIMO-OFDM systems, which has been originally proposed in [7] for the case of single carrier. This generalizes the idea of pilot-symbol-assisted modulation (PSAM) and pilot-embedding techniques.

The organization of this paper is as follows. In section II, we briefly introduce the wireless channel and system models used in our paper. In section III, we propose the pilot-embedded data-bearing approach for joint channel estimation and data detection including the basic LS channel estimation and ML data detection. The FFT-based and adaptive FFT-based channel estimations are considered in section IV. In section $V$, the performance evaluation by computer simulations is examined, and the conclusion is given in section VI. For ease of later use, let $(\cdot)^{T}$ stands for the transpose of a matrix, $(\cdot)^{H}$ stands for the complex-conjugate transpose of a matrix, $\mathbf{I}$ stands for an identity matrix, and $\mathbf{0}$ stands for an all-zero-element matrix.

\section{Wireless Channel And System Models}

In this section, we introduce the wireless channel and system models used in this paper. A $K$-tone SF-coded OFDM system with $L_{r}$ receive and $L_{t}$ transmit antennas is considered.

\section{A. Wireless Channel Model}

The complex baseband impulse response of the wireless channel between the $a^{t h}\left(a=1, \ldots, L_{r}\right)$ receive antenna and 
the $b^{\text {th }}\left(b=1, \ldots, L_{t}\right)$ transmit antenna can be described by

$$
h_{a b}(t, \tau)=\sum_{l} \gamma_{l}(t) \delta\left(\tau-D_{l}\right)
$$

where $D_{l}$ is the delay of the $l^{t h}$ path and $\gamma_{l}(t)$ represents the corresponding complex amplitude. $\gamma_{l}(t)$ 's are modelled as wide-sense stationary (WSS), narrowband complex Gaussian processes, which are independent for different paths, and $\mathrm{E}\left[\left|\gamma_{l}(t)\right|^{2}\right]=\sigma_{l}^{2}$ with $\sigma_{l}^{2}$ being an average power of the $l^{\text {th }}$ path. Throughout this paper, we assume that all the signals transmitted from different transmit antennas and received at different receive antennas undergo independent fades, and the channel average power is normalized to have $\sum_{l} \sigma_{l}^{2}=1$. For OFDM systems with tolerable leakage, the normalized frequency response of the OFDM systems at the $k^{\text {th }}(k=0, \ldots, K-1)$ subcarrier between the $a^{\text {th }}$ receive antenna and the $b^{\text {th }}$ transmit antenna can be described by [5]

$$
H_{a b}(m, n, k)=\sum_{l=0}^{L-1} h_{a b}(m, n, l) F_{K}^{k \tau_{l}},
$$

where $h_{a b}(m, n, l) \triangleq h_{a b}\left(m, n T_{f}, \tau_{l} t_{s}\right), \quad F_{K}=$ $\exp (-j 2 \pi / K), \quad t_{s}=1 / K \Delta f$, with $\Delta f$ being the tone spacing, is the sample interval of the system, $T_{f}$ is the OFDM block length, and $m$ denotes index of a group of $N$-OFDM blocks described next. $L$ is the number of nonzero paths, which represents the order of frequency diversity of the channel, and $\tau_{l}(l=0, \ldots, L-1)$ is the $l^{t h}$ path's delay sampled at a rate of $1 / t_{s}$. Furthermore, the average power of $h_{a b}(m, n, l)$ and the value of $L(\ll K)$ depend on the delay profile and the dispersion of the wireless channels. For ease of later use, we omit the time index $n$ in all parameters.

\section{B. System Model}

At the transmitter side, the data stream is split into $L_{t}$ substreams, and, in each substream, a group of data is chosen to match to the corresponding baseband $M$-phase-shift keyed (MPSK) constellation symbol. These MPSK-data symbols are then coded by the SF block code, e.g. [3], and grouped to construct the $L_{t} \times K N$ SF-coded data matrix $\mathbf{S}(m)$, where $N$ denotes the number of OFDM blocks (each OFDM block has $K$ tones) to be regarded as one SF-coded data block, and $m$ denotes the $N$-OFDM-block index. Before modulating this SFcoded data block by the OFDM modulator, the SF-coded data matrix $\mathbf{S}(m)$ is embedded by the pilot signal using the pilotembedded data-bearing approach resulting in the $L_{t} \times K M$ SFcoded symbol matrix, where $M$ denotes the number of OFDM blocks to be regarded as one SF-coded symbol block. Notice that $M>N$ in which the redundancy is introduced after embedding the pilot signal for acquiring the CSI. Each pilotembedded OFDM block is then modulated and simultaneously transmitted across $L_{t}$ transmit antennas. In order to eliminate the intersymbol interference (ISI), we employ a cyclic prefix in which the length of cyclic extension must be larger than or, at least, equal to $\tau_{L-1}$. In this paper, we consider two types of fading channels: quasi-static and nonquasi-static frequencyselective Rayleigh fading channels. The former is the scenario that the channel remains constant over the SF-coded symbol block but changes in block-by-block basis, whereas in the latter the channel changes within the SF-coded symbol block. At the receiver side, the received signal is sampled at rate $t_{s}$ and demodulated by the OFDM demodulator. By assuming tolerable power leakage and perfect time/frequency synchronization, the reconstructed signal of the $m^{\text {th }} \mathrm{SF}$-coded symbol block can be described by

$$
\mathbf{Y}(m)=\mathbf{H}(m) \mathbf{U}(m)+\mathbf{Z}(m),
$$

where $\mathbf{Y}(m)$ is the $L_{r} \times K M$ received signal matrix; $\mathbf{H}(m)$ is the $L_{r} \times K L_{t}$ channel matrix in which the $a^{t h}$ row of $\mathbf{H}(m)$ is $\left[\mathbf{H}_{a 1}(m), \ldots, \mathbf{H}_{a L_{t}}(m)\right]$ where $\mathbf{H}_{a b}(m)=$ $\left[H_{a b}(m, 0), \ldots, H_{a b}(m, K-1)\right] ; \mathbf{Z}(m)$ is the $L_{r} \times K M$ additive white Gaussian noise (AWGN) matrix with zero mean and variance $\frac{\sigma^{2}}{2} \mathbf{I}_{\left(L_{r} K M \times L_{r} K M\right)}$ per real dimension; and $\mathbf{U}(m)$ is the $K L_{t} \times K M$ equivalent SF-coded symbol matrix. Throughout this paper, we assume that the channels and noise, and channels from different paths are mutually uncorrelated.

\section{The Pilot-Embedded Data-Bearing APProach FOR JoINT ChanNEl ESTIMATION AND DATA DETECTION}

In this section, we first propose the pilot-embedded databearing approach for joint channel estimation and data detection, then we briefly introduce the LS channel estimation and the ML data detection. In the pilot-embedded data-bearing approach for joint channel estimation and data detection, the equivalent SF-coded symbol matrix $\mathbf{U}(m)$ can be described as follows,

$$
\mathbf{U}(m)=\mathbf{D}(m) \mathbf{A}+\mathbf{P},
$$

where $\mathbf{D}(m)$ denotes the $K L_{t} \times K N$ equivalent SF-coded data matrix constructed from the $L_{t} \times K N$ SF-coded data matrix $\mathbf{S}(m)$ using the $K \times K$ matrix-diagonalized operator $\operatorname{diag}\{\cdot\}$, where the $((b-1) K+1)^{t h}$ row to the $(b K)^{\text {th }}$ row of $\mathbf{D}(m)$ are $\left[\operatorname{diag}\left\{[\mathbf{S}(m)]_{b, 1: K}\right\}, \ldots, \operatorname{diag}\left\{[\mathbf{S}(m)]_{b,(N-1) K+1: N K}\right\}\right]$ with $x: y$ denotes the column/row index interval ranging from column/row $x$ to column/row $y ; \mathbf{A}$ is the $K N \times K M$ data bearer matrix; and $\mathbf{P}$ is the $K L_{t} \times K M$ pilot matrix. Notice that the $K$ diagonal elements of a $(b, c)^{t h}$ submatrix, $c=1, \ldots, M$, constructed by splitting $\mathbf{U}(m)$ from the $((b-1) K+1)^{t h}$ row to the $(b K)^{t h}$ row and the $((c-1) K+1)^{t h}$ column to the $(c K)^{\text {th }}$ column are the $c^{t h}$ transmitted SF-coded OFDM block of the $b^{t h}$ transmit antenna in the $m^{\text {th }}$ SF-coded symbol block. In addition, the energy constraint $\mathrm{E}\left[\|\mathbf{D}(m)\|^{2}\right]=K L_{t}$ is maintained for the equivalent SF-coded data matrix. Substituting (4) into (3), the received signal matrix can be rewritten as

$$
\mathbf{Y}(m)=\mathbf{H}(m)(\mathbf{D}(m) \mathbf{A}+\mathbf{P})+\mathbf{Z}(m) .
$$

Now, by the pilot-embedded data-bearing approach, we mean that the data bearer matrix $\mathbf{A}$ and the pilot matrix $\mathbf{P}$ satisfy the following properties:

$$
\begin{gathered}
\mathbf{A} \mathbf{P}^{T}=\mathbf{0}_{\left(K N \times K L_{t}\right)}, \mathbf{P} \mathbf{P}^{T}=\alpha \mathbf{I}_{\left(K L_{t} \times K L_{t}\right)}, \\
\mathbf{P A}^{T}=\mathbf{0}_{\left(K L_{t} \times K N\right)}, \text { and } \mathbf{A} \mathbf{A}^{T}=\beta \mathbf{I}_{(K N \times K N)},
\end{gathered}
$$

where $\beta$ is the real-valued data-power factor, $\alpha$ is the realvalued pilot-power factor. The similar property $\mathbf{P P}^{T}=\alpha \mathbf{I}$ in (6) is also suggested in [6] that they are the optimal criteria for the optimal training design for MIMO OFDM systems. There are many possible structures of data-bearing and pilot matrices, 
in a similar way to [7], that satisfy the properties (6) and (7). However, we are interested in the Code-Multiplexing (CM)based structure because it is the best among all other structures [7], given as

$$
\begin{gathered}
\mathbf{A}=\sqrt{\beta} \mathrm{WH}[1: N]_{(N \times M)} \otimes \mathbf{I}_{(K \times K)}, M=N+L_{t}, \\
\mathbf{P}=\sqrt{\alpha} \mathbf{W H}[N+1: M]_{\left(L_{t} \times M\right)} \otimes \mathbf{I}_{(K \times K)},
\end{gathered}
$$

where $\mathrm{WH}[x: y]$ denotes a sub-matrix created by splitting the $M \times M$ normalized Walsh-Hadamard matrix starting from $x^{t h}$-row to $y^{t h}$-row. The disadvantage of this structure is the limitation of dimensionality of Walsh-Hadamard matrix, which has a dimension proportionally to $2^{n}, n \in \mathbb{I}$. In addition, this structure provides an instructive example of the proposed general idea in (4) for pilot-embedding.

\section{A. LS Channel Estimation}

We first extract the pilot part from the received signal matrix $\mathbf{Y}(m)$. By the virtue of null-space and orthogonality properties in (6), respectively, we are able to extract the pilot part by simply post multiply $\mathbf{Y}(m)$ in (5) by $\mathbf{P}^{T}$, and divide the result by $\alpha$, to arrive at

$$
\frac{\mathbf{Y}(m) \mathbf{P}^{T}}{\alpha}=\mathbf{H}(m)+\frac{\mathbf{Z}(\mathbf{m}) \mathbf{P}^{T}}{\alpha} .
$$

Let $\mathbf{Y}_{1}(m)=\frac{\mathbf{Y}(m) \mathbf{P}^{T}}{\alpha}$ and $\mathbf{Z}_{1}(m)=\frac{\mathbf{Z}(\mathbf{m}) \mathbf{P}^{T}}{\alpha}$, we have

$$
\mathbf{Y}_{1}(m)=\mathbf{H}(m)+\mathbf{Z}_{1}(m)
$$

It can be shown that the LS channel estimator can be described by

$$
\hat{\mathbf{H}}_{L S}(m)=\mathbf{Y}_{1}(m)=\frac{\mathbf{Y}(m) \mathbf{P}^{T}}{\alpha} .
$$

\section{B. ML Data Detection}

we now explain the procedure of ML data detection. First, we extract the data part from the received signal matrix $\mathbf{Y}(\mathrm{m})$. By the virtue of null-space and orthogonality properties in (7), respectively, we are able to extract the data part by simply post multiply $\mathbf{Y}(m)$ in (5) by $\mathbf{A}^{T}$, and divide the result by $\beta$, to arrive at

$$
\frac{\mathbf{Y}(m) \mathbf{A}^{T}}{\beta}=\mathbf{H}(m) \mathbf{D}(m)+\frac{\mathbf{Z}(\mathbf{m}) \mathbf{A}^{T}}{\beta} .
$$

Let $\mathbf{Y}_{2}(m)=\frac{\mathbf{Y}(m) \mathbf{A}^{T}}{\beta}$ and $\mathbf{Z}_{2}(m)=\frac{\mathbf{Z}(\mathbf{m}) \mathbf{A}^{T}}{\beta}$, we have

$$
\mathbf{Y}_{2}(m)=\mathbf{H}(m) \mathbf{D}(m)+\mathbf{Z}_{2}(m) \text {. }
$$

From the orthogonality of $\mathbf{A}$ in (7) and $\sum_{j^{\prime}=1}^{K M}\left|A_{i^{\prime}, j^{\prime}}\right|^{2}=$ $\beta, \forall i^{\prime}$, which is implied from (7), the data-bearer-projected noise $\mathbf{Z}_{2}(m)$ is the zero mean AWGN with variance $\frac{\sigma^{2}}{2 \beta} \mathbf{I}_{\left(K L_{r} N \times K L_{r} N\right)}$ per real dimension. Due to the i.i.d white Gaussian distribution of $\mathbf{Z}_{2}(m)$, the ML receiver for the $d^{\text {th }}$ OFDM block in the $m^{\text {th }}$ SF-coded data block transmitted from the $b^{\text {th }}$ transmit antenna decides the codeword that minimizes the decision matric given by

$$
\begin{gathered}
\hat{\mathbf{D}}_{i, j}(m)=\min _{\mathbf{D}_{i, j}}\left\|\mathbf{Y}_{2_{s, j}}(m)-\hat{\mathbf{H}}_{s, i}(m) \mathbf{D}_{i, j}(m)\right\|^{2}, \\
i=(b-1) K+1: b K, j=(d-1) K+1: d K, \\
s=1: L_{r}, \text { and } d=1, \ldots, N,
\end{gathered}
$$

where $\hat{\mathbf{H}}(m)$ is the estimated channel matrix, e.g. $\hat{\mathbf{H}}(m)=$ $\hat{\mathbf{H}}_{L S}(m)$.

\section{The Proposed Adaptive FFT-Based Channel ESTIMATION}

In this section, we improve the performance of the LS channel estimator in (11) by employing the FFT-based approach proposed in [5]. First, we briefly summarize the concept of the FFT-based channel estimator and point out an inherent problem. Then we propose the adaptive FFT-based channel estimation.

\section{A. FFT-Based Channel Estimation Approach}

As suggested in [5], the FFT-based channel estimation approach firstly performs the LS channel estimation by using the $L$ significant taps, and IFFT transformation. The resulting temporal LS channel estimate is then FFT transformed to the $K$ subcarrier channel frequency response. The simplified approach is also suggested in [5] by choosing $P$ significant taps, in the sense that $P^{\prime}$ 's largest $\sum_{b=1}^{L_{t}}\left|\hat{H}_{L S_{a b}}(m, k)\right|^{2}, k=0, \ldots, K-1$ is selected, instead of using $L$ significant taps.

Now let us describe the FFT-based channel estimation in details. From (11), we have $\hat{\mathbf{H}}_{L S_{a b}}(m)=$ $\left(\left[\hat{\mathbf{H}}_{L S}\right]_{a,(b-1) K+1: b K}\right)^{T}$. From the channel model in (2), $\hat{\mathbf{H}}_{L S_{a b}}(m)$ can be expressed as

$$
\hat{\mathbf{H}}_{L S_{a b}}(m)=\mathbf{F h}_{a b}(m)+\mathbf{Z}_{1_{a b}}(m),
$$

where $\mathbf{F}$ is the $K \times L$ matrix whose element $[\mathbf{F}]_{x y}$ is defined by $\exp (-j 2 \pi / K)(x-1) \tau_{y}, x=1, \ldots, K, y=0, \ldots, L-1$; $\mathbf{h}_{a b}(m)=\left[h_{a b}(m, 0), \ldots, h_{a b}(m, L-1)\right]^{T} ;$ and $\mathbf{Z}_{1_{a b}}(m)=$ $\left(\left[\mathbf{Z}_{1}(m)\right]_{a,(b-1) K+1: b K}\right)^{T}$. Notice that the LS channel estimate in (15) represents the $K$-tap FFT-based channel estimate.

Transforming the LS channel estimate in (15) to the temporal LS channel estimate by using the $K \times K$ IFFT matrix $\mathbf{G}$, whose element $[\mathbf{G}]_{x y}$ is defined by $\frac{1}{K} \exp (j 2 \pi / K)(x-1)(y-$ 1), $x, y=1, \ldots, K$ to arrive at

$$
\hat{\mathbf{h}}_{L S_{a b}}(m)=\mathbf{G H}_{L S_{a b}}(m)=\mathbf{G F h}_{a b}(m)+\mathbf{G Z}_{1_{a b}}(m) \text {. }
$$

It can be shown that

$$
\begin{gathered}
{[\mathbf{G F}]_{x y}=\frac{f\left(x-1-\tau_{y}\right)}{K} \exp \left(j \xi\left(x-1-\tau_{y}\right)\right),} \\
x=1, \ldots, K, y=0, \ldots, L-1,
\end{gathered}
$$

where $f(q)=\frac{\sin (\pi q)}{\sin (\pi q / K)}$ is the leakage function and $\xi=$ $\frac{(K-1) \pi}{K}$. Note that if $q$ is equal to an integer number, then $f(q)=0$; if $q$ is equal to zero, then $f(0)=K$. Substituting (17) into the first term on the r.h.s. of (16) to arrive at

$$
\begin{gathered}
\mathbf{G F h}_{a b}(m)=\frac{1}{K}\left[\sum_{l=0}^{L-1} h_{a b}(m, l) f\left(-\tau_{l}\right) e^{j \xi\left(-\tau_{l}\right)}, \ldots,\right. \\
\left.\sum_{l=0}^{L-1} h_{a b}(m, l) f\left(K-1-\tau_{l}\right) e^{j \xi\left(K-1-\tau_{l}\right)}\right]^{T} .
\end{gathered}
$$

Let $\mathbf{Z}_{1_{a b}}^{G}(m)=\mathbf{G} \mathbf{Z}_{1_{a b}}(m)$, then substituting $\mathbf{Z}_{1_{a b}}^{G}(m)$ and (18) into (16) given by

$$
\hat{\mathbf{h}}_{L S_{a b}}(m)=[g(1), \ldots, g(K)]^{T},
$$

where $g(x)=\frac{1}{K} \sum_{l=0}^{L-1} h_{a b}(m, l) f\left(x-1-\tau_{l}\right) e^{j \xi\left(x-1-\tau_{l}\right)}+$ $Z_{1_{a b}}^{G}(m, x)$ with $Z_{1_{a b}}^{G}(m, x)$ being the $x^{\text {th }}$ element of $\mathbf{Z}_{1_{a b}}^{G}(m)$.

Obviously, from (19), if $\tau_{l}$ is an integer number, then the $l^{\text {th }}$ element of the $L$ largest elements of $\hat{\mathbf{h}}_{L S_{a b}}(m)$ is equal to $h_{a b}(m, l)+Z_{1_{a b}}^{G}(m, l)$, and the rest elements, which are not a member of the $L$ largest elements, are equal to $Z_{1_{a b}}^{G}(m, e), e \neq$ $l, e \in\{0, \ldots, K-1\} \backslash \mathcal{W}$ with $\mathcal{W}$ being the set of the $L$ 
largest elements. As a result, by choosing the $L$ largest taps and replacing the $(K-L)$ remaining taps by zero is sufficient and optimal for constructing the FFT-based estimated temporal channel impulse response $\hat{\mathbf{h}}_{F F T_{a b}}(m)$, since we completely capture the channel impulse response $\mathbf{h}_{a b}(\mathrm{~m})$, and delete the excessive noise in the $(K-L)$ remaining taps out. However, in reality, the $l^{t h}$ multipath delay $\tau_{l}$ is a non-integer number, hence, the $L$-multipath channel impulse response dissipates to all $K$ taps of $\hat{\mathbf{h}}_{L S_{a b}}(m)$ resulting in the model mismatch error that increases the channel estimation error, primarily caused by the AWGN $\mathbf{Z}_{1_{a b}^{G}}^{G}(m)$. This additional channel estimation error causes the severe error floor in the mean square error (MSE) of the channel estimation, and the detection error probability. Once the $L$ or $P$ largest taps are chosen and the rest taps are replaced by zero, the FFT-based estimated channel frequency response is determined by

$$
\hat{\mathbf{H}}_{F F T_{a, b}}(m)=K \mathbf{G}^{H} \hat{\mathbf{h}}_{F F T_{a b}}(m) .
$$

In what follows, we propose the alternative approach for FFTbased channel estimation to overcome the model mismatch error.

\section{B. The Proposed Adaptive FFT-Based Channel Estimation}

The model mismatch error in the FFT-based channel estimation stems from the fact that a fixed number of $L$ or $P$ largest taps is used in the channel estimation process for all signal-tonoise ratio (SNR) values. This has been suggested in [5] that, in low SNR regimes, the channel estimation error is mainly caused by the AWGN, hence, a small number of taps is recommended in order to reduce the noise effect; as a result, a lower channel estimation error is obtained. In high SNR regimes, the channel estimation error is mainly caused by the model mismatch error, hence, a large number of taps is suggested in order to compensate this model mismatch error; as a result, the enhanced channel estimation error is obtained. Based on this basic idea, we now propose the adaptive FFT-based channel estimation as follows. We propose that the number of significant taps $P_{\text {opt }}$ used to capture the CSI in $\hat{\mathbf{h}}_{L S_{a b}}(m)$ in (19) is obtained by solving the following optimization problem:

$$
\begin{gathered}
P_{o p t}=\min (P) \text { s.t. } \\
\sum_{a=1}^{L_{r}} \sum_{b=1}^{L_{t}} \sum_{j=1}^{P} \mathrm{E}\left[\max _{i_{j} \in\{1, \ldots, K\}}\left\|\left[\hat{\mathbf{h}}_{L S_{a b}}(m)\right]_{i_{j}}\right\|^{2}\right] \geq \\
\sum_{a=1}^{L_{r}} \sum_{b=1}^{L_{t}} \sum_{j=1}^{K} \mathrm{E}\left[\left\|\left[\hat{\mathbf{h}}_{L S_{a b}}(m)\right]_{j}\right\|^{2}\right]-\frac{(K-P) L_{t} L_{r} \sigma^{2}}{K \alpha}= \\
\sum_{a=1}^{L_{r}} \sum_{b=1}^{L_{t}} \sum_{j=1}^{K} \sum_{l=0}^{L-1} \mathrm{E}\left[\left|h_{a b}(m, l) f\left(j-1-\tau_{l}\right)\right|^{2}\right]+\frac{P L_{t} L_{r} \sigma^{2}}{K \alpha},
\end{gathered}
$$

where the second equality comes from the assumption that the channel $h_{a b}(m, l)$ and noise are mutually uncorrelated. In addition, $\sum_{a=1}^{L_{r}} \sum_{b=1}^{L_{t}} \sum_{j=1}^{K} \mathrm{E}\left[\left\|\left[\mathbf{Z}_{1_{a b}^{G}(m)}\right]_{j}\right\|^{2}\right]=\frac{L_{t} L_{r} \sigma^{2}}{\alpha}$. The detail derivation of $(21)$ is omitted here due to the page limitation.

Intuitively explaining, this optimization approach is to find the minimum number of significant taps that can capture the average total energy of the channel dissipating in all $K$ taps in the presence of AWGN. Notice that, the optimum number of taps increases as the noise variance decreases (i.e. the SNR increases). In high SNR regimes, the model mismatch error approaches to zero. This phenomenon stems from the fact that the constraint in (21) will exist if a large number of taps are used, due to the small variance of AWGN. In low SNR regimes, the small number of taps is resulted, as we expected, since the variance of AWGN is large resulting in the small number of taps is enough to make the constraint in (21) existed.

\section{Computer Simulations}

To illustrate the performance of the proposed scheme, simulations are conducted under two different scenarios: quasi-static and nonquasi-static frequency-selective Rayleigh fading channels. The simulated SF block code is obtained from Alamouti's structure, as proposed in [3], whose elements are taken from a BPSK constellation for two transmit antennas and two receive antennas. The COST207 typical urban (TU) six-ray power delay profile [5] with delay spread of $5 \mu s$ is studied. The entire channel bandwidth, $1 \mathrm{MH}_{z}$, is divided into $K=128$ subcarriers in which four subcarriers on each end are served as guard tones, and the rest (120 tones) are used to transmit data. To make the tone orthogonal to each other, the symbol duration is $128 \mu \mathrm{s}$, and additional $20 \mu \mathrm{s}$ guard interval is used as the cyclic prefix length in order to protect the ISI due to the multipath delay spread. This results in a total block length $T_{f}=148 \mu \mathrm{s}$ and a subchannel symbol rate $r_{b}=6.756 \mathrm{KBd}$. In addition, the equal block-power allocation, i.e. $\beta=\alpha=0.5 \mathrm{~W}$, is employed, and the normalized SF-coded symbol block-power is $1 \mathrm{~W}$.

\section{A. Quasi-Static Channel Scenario}

In this scenario, the channel impulse response $h_{a b}(m, l)$ 's in (2) are from the normalized time-varying channel which is modelled as Jake's model [8], when $f_{d} * T_{f}=0.08$ (fast fading) with $f_{d}$ being the Doppler's shift.

In Fig. 1 and 2, the MSEs and BERs of the LS, 10-tap FFTbased, adaptive FFT-based, and LMMSE channel estimators [4] are shown, respectively. Notice that the LS channel estimator has higher MSE and BER than that of the 10-tap FFT-based and the adaptive FFT-based channel estimators in low SNR regimes. In high SNR regimes, the LS and adaptive FFT-based channel estimators performs better than the 10-tap FFT-based channel estimator in which the error floor caused by the model mismatch error occurs. It is worth noticing that the LMMSE channel estimator serves as a performance bound.

\section{B. Nonquasi-Static Channel Scenario}

For the sake of exposition, we study a 4-block fading model in which the channel impulse response $h_{a b}(m, l)$ symmetrically changes four times within one SF-coded symbol block, i.e. there exists $\mathbf{H}_{1}(m)$ to $\mathbf{H}_{4}(m)$ in the $m^{\text {th }}$-block SF-coded symbol matrix. In Fig.3, the BERs of the SF-coded MIMO-OFDM system employing the LS, 10-tap FFT-based, adaptive FFTbased, and LMMSE channel estimators and $f_{d} * T_{f}$ are 0.04 and 0.064 , are shown. Notice that when the Doppler's shift is small $\left(f_{d} * T_{f}=0.04\right)$ in high SNR regimes, the 10-tap FFT-based channel estimator performs the worst. In low SNR regimes, the LS channel estimator performs the worst. When Doppler's shift is high $\left(f_{d} * T_{f}=0.064\right)$ in high SNR regimes, all 


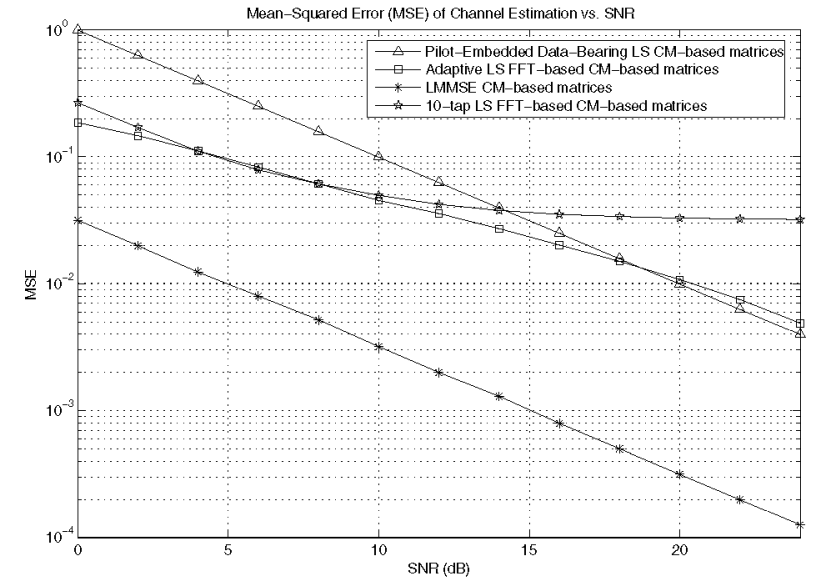

Fig. 1: The graph of MSEs of the channel estimation in quasi-static fading channels.

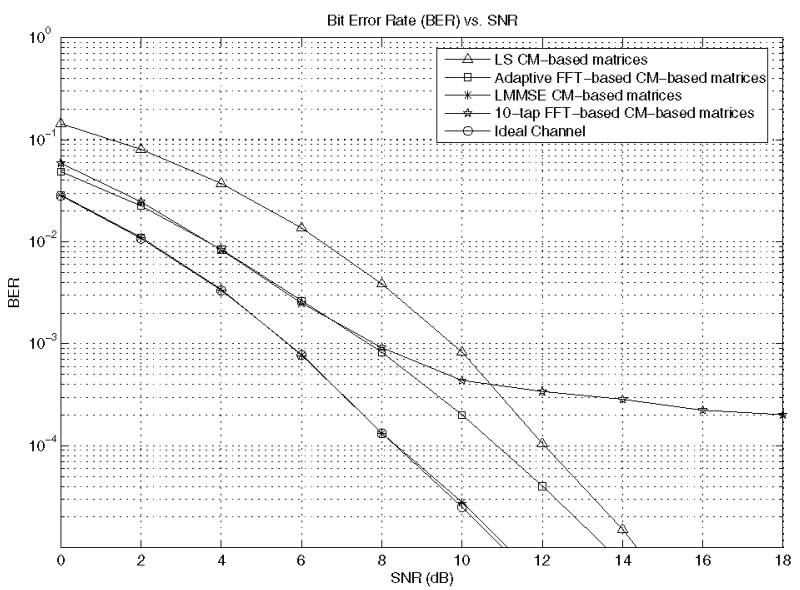

Fig. 2: The graph of BERs of the pilot-embedded SF-coded MIMOOFDM system in quasi-static fading channels.

channel estimators yields quite close results. This phenomenon stems from the fact that the channel mismatch error dominates all factors causing the detection error. In Fig.4, the optimum number of significant taps for both quasi-static and nonquasistatic fading channels is shown.

\section{CONCLUSION}

In this paper, we have proposed the adaptive FFT-based channel estimator for improving the performances of the FFT-based and LS channel estimators, and the pilot-embedded data-bearing approach for joint channel estimation and data detection. Simulations were conducted to examine the performance of the proposed scheme. For quasi-static (and nonquasi-static with low Doppler's shifts, under high SNR regimes) TU-profile fading channels, the adaptive FFT-based channel estimator performs the best. Furthermore, for nonquasi-static fading channels with high Doppler's shifts, the channel mismatch error dominates all factors causing the detection error, and thus results in comparable error floors for all channel estimators.

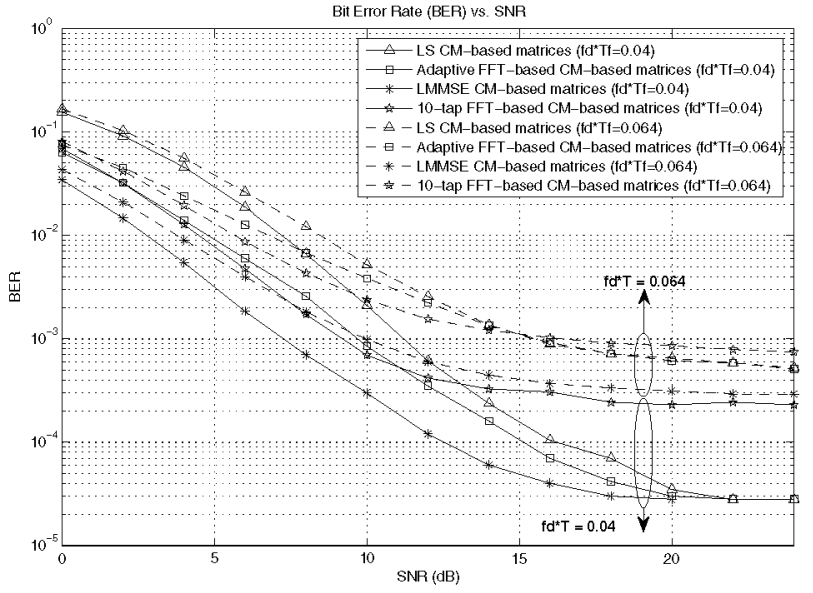

Fig. 3: The graph of BERs of the pilot-embedded SF-coded MIMOOFDM system in nonquasi-static fading channels.

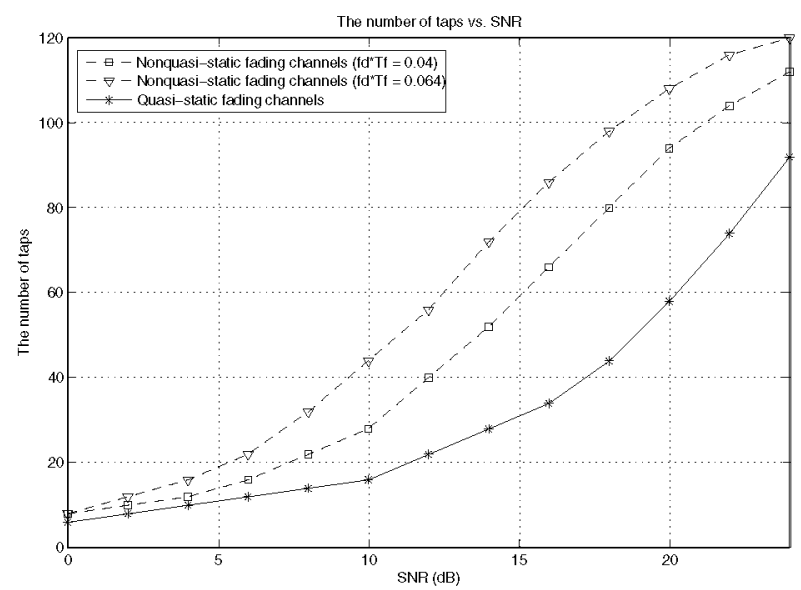

Fig. 4: The graph of the optimum number of significant taps for both quasi-static and nonquasi-static TU-profile fading channels.

\section{REFERENCES}

[1] T. Ojanperä and R. Prasad, "An overview of third generation wireless personal communications: A european perspective," IEEE Pers. Commu., Vol. 5, pp. 59-65, Dec. 1998

[2] L.J. Cimini, Jr. and N.R. Sollenberger, "OFDM with diversity and coding for high-bit-rate mobile data applications," in Proc. IEEE Globecom, Pheonix, AZ, pp. 305-309, Nov. 1997.

[3] Weifeng Su, Z. Safar, M. Olfat, and K.J.R. Liu, "Obtaining full-diversity space-frequency codes from space-time codes via mapping," IEEE Trans. Signal Processing, Vol. 51, No. 11, pp. 2905-2916, Nov. 2003.

[4] Y. Gong and K.B. Lataief, "Low complexity channel estimation for spacetime coded wideband OFDM systems," IEEE Trans. Wireless Commu., Vol. 2, No. 5, pp. 876-882, Sept. 2003.

[5] Y. (Geoffrey) Li, "Channel estimation for OFDM systems with trsmitter diversity in mobile wireless channels," IEEE J. Select. Areas Commu., Vol. 17, No. 3, pp. 461-471, Mar. 1999.

[6] Imad Barhumi, G. Leus, and M. Moonen, "Optimal training design for MIMO OFDM systems in mobile wireless channels," IEEE Trans. Signal Processing, Vol. 51, No. 6, pp. 1615-1624, June 2003.

[7] C. Pirak, Z.J. Wang, K.J.R. Liu, and S. Jitapunkul, "Performance analysis for pilot-embedded data-bearing approach in space-time coded MIMO systems," in Proc. ICASSP, Philadelphia, PA, pp.593-596, Mar. 2005.

[8] W.C. Jakes, Jr., "Multipath Interference", in Microwave Mobile Communication W.C. Jakes, Jr., Ed., Wiley, NY, 1974, pp. 67-68. 